\title{
Convention on the rights of persons with disabilities: Qualitative exploration of barriers to the implementation of articles 25 (health) and 26 (rehabilitation) in Iran
}

\author{
Zahra Najafi ${ }^{1}$, Kianoush Abdi*1 (D), Mohammad Saeed Khanjani ${ }^{2}$, Hamid Dalvand $^{3}$, Mehdi Amiri $^{1}$ \\ Received: 4 Feb 2020 \\ Published: 19 Jan 2021
}

\section{Abstract}

Background: The International Convention on the Rights of Persons with Disabilities (CRPD) is the most important International Document for recognizing the rights of persons with disabilities, including the right to health and rehabilitation. Islamic Republic Iran acceded to the Convention in 2008, but still has a long way to go to achieve its desired status and in line with the objectives of the convention. This study aimed to identify the barriers to the implementation of articles 25 and 26 of the CRPD in Iran.

Methods: This study was performed using conventional content analysis. Twenty-one individuals were recruited by purposive sampling with maximum variation and were continued until saturation. Data were gathered through in-depth, semi-structured interviews from June 2018 to May 2019. MAXQDA version 10 was used for analyzing data.

Results: The resulting data analysis yielded 860 initial or open codes. The concepts were categorized into 27 subcategories and 7 categories. Main categories were included: "Structure inefficiency", "lack of comprehensive rehabilitation program", "inadequate awareness", "neglected economy of people with disabilities", "weak access to services", "cultural challenges" and "disregard for new technologies".

Conclusion: The findings showed that the executive structures in the country have a lot of problems with health and rehabilitation programs for people with disabilities. It seems understanding the barriers to implementation of articles 25 and 26 of the international CRPD empowers officials in the field and improve services by providing a better view of the disabled. Nevertheless, it is recommended for policymakers to consider rehabilitation as a main element of the health system.

Keywords: Convention on the Rights, Disabilities, Health, Rehabilitation, Content analysis

Conflicts of Interest: None declared

Funding: University of Social Welfare \& Rehabilitation Sciences (grant No. 2145)

\section{*This work has been published under CC BY-NC-SA 1.0 license. \\ Copyright $\odot$ Iran University of Medical Sciences}

Cite this article as: Najafi Z, Abdi K, Khanjani MS, Dalvand H, Amiri M. Convention on the rights of persons with disabilities: Qualitative exploration of barriers to the implementation of articles 25 (health) and 26 (rehabilitation) in Iran Med J Islam Repub Iran. 2021 (19 Jan);35:10. https://doi.org/10.47176/mjiri.35.10

Corresponding author: Dr Kianoush Abdi, ki.abdi@uswr.ac.ir

Department of Rehabilitation Management, University of Social Welfare and Rehabilitation Sciences, Tehran, Iran

2. Department of Counseling, University of Social Welfare and Rehabilitation Sciences, Tehran, Iran

3. Department of Occupational Therapy, School of Rehabilitation, Tehran University of Medical Sciences, Tehran, Iran. $\uparrow$ What is "already known" in this topic:

Countries in the West Asian region, including Iran, have a large number of PWD due to natural disasters, hereditary diseases, and warfare. The experiences of PWD show that despite the ratification of the International Convention, their rights to promote and enforce human rights, especially in developing countries, remain an important challenge to the implementation of Articles 25 and 26 of the Disability Rights Convention.

\section{$\rightarrow$ What this article adds:}

Our results of this study will provide a clear pathway for managers, planners rehabilitation for the PWD to be able to reform their executive structures and to develop comprehensive programs, like other ordinary people, and help them in achieving their human rights in health and rehabilitation The findings could lead to a change in health policymakers' attitudes toward rehabilitation services as one of the rights of PWD. 


\section{Introduction}

According to the report of the World Health Organization (WHO), about $15 \%$ of the world's population suffers from various types of disabilities (1), while governmental resources in Iran account for about 4\% of the population (2). At every point in history, societal approaches to disability varied: sometimes, like primitive communities, people with disabilities (PWD) were destroyed at birth to avoid overburdening, and sometimes they were treated with pity, and they were considered worthy of kindness and charity (3).

Today also PWD have many social limitations in different societies, because they are more likely to miss primary educations, receive a less formal education, and are less likely to find a job, earnings, and it is more difficult to access public services and are subject to discrimination (4). However, today neither society nor the PWD accepts these views, and they, rather than seeking special privileges, want to play equal roles and responsibilities with other members of society and organize powerful organizations in pursuit of their neglected rights (3). In fact, disability is a human rights issue because PWD experience inequalities simply because of their disability. For example, they are deprived of equal access to health care, employment, education, and political participation. They are also often abused and degraded (1).

The International CRPD is an international document for the promotion of the rights of persons with disabilities approved on December 13, 2006, at the United Nations General Assembly with fifty articles covering various aspects of their lives, including civil, political, cultural, and social rights (5). The Convention is the first document that guarantees the rights of PWD with particular concerns (6), puts their problems on the global agenda, and calls for their greater participation in society as citizens with dignity. The International CRPD as a law redefines the world and the living status of PWD (7).

Article 25 of the Convention clearly states the right to the highest acceptable level of health without discrimination due to disability (5). This right is further extended in Article 26 and obliged states, including the support of peer groups, to enable PWD to achieve their maximum independence and maintain physical, mental, social, and professional abilities, as well as full participation in all aspects of lives (5).

Paragraph E of Article 25 of the Convention states that health insurance and life insurance should be provided in a fair and non-discriminatory manner to PWD (5). Some studies show that few women with disabilities are covered by the health insurance and there is a significant difference in the quality of care between the two subgroups of insurance (8). Article 25 of the Convention also states that for PWD the same quality and free and payable standards of health care are provided as to the others, including terms of sexual and reproductive health and populationbased health programs (5).

Article 26 states that governments are obliged to organize, strengthen, and extend services and facilitation the rehabilitation programs, especially in the field of health
(5). According to Article 25, in fulfilling their obligations under the Convention, the states must provide health and rehabilitation services in an appropriate manner to the sex, the need, and the age of the person $(9,10)$.

The experiences of PWD show that despite the ratification of the International Convention, their rights to promote and enforce human rights, especially in developing countries, remain an important challenge(4). Abdi's in his study (2015) explaining the challenges of providing rehabilitation services to PWD in Iran, identified six categories of challenges including lack of knowledge, negative attitudes toward disability, inadequate support for PWD, problems for the providers, access problems, and payment costs (11).

Countries in the West Asian region, including Iran, have a large number of PWD due to natural disasters, hereditary diseases, and brutal warfare. Such states appear to be able to resolve their problems in their cooperation with the CRPD (Article 12) and the General Assembly (3).

Iran joined the International CRPD in 2008 (12). In the Islamic Republic of Iran, despite the value-based attitude of Islamic worldview and despite some interesting and beautiful points in Islamic texts including the Holy Qur'an and its various interpretations, the Al-sira al-Nabawiyya (Prophetic biography) the Imams Sira, and jurisprudential, legal, theological, historical, and literary books (13), and with respect to being underdeveloped, the right-centered approach to PWD has not received much attention and has only been formulated and enacted in a variety of laws and regulations specific to $\mathrm{PWD}$, with a view to being supportive-centered. For various reasons, it has not been effective in achieving the intended objectives set forth in the constitution and even in religious doctrine (14).

The higher the quality and rate of health indicators in a society and the more balanced and appropriate the distribution of these indicators, the better the relative wellbeing and health of that community $(15,16)$. It should also be considered that the health system is one of the major parts of any country that is affected by the country's economy and its determinants (17). Statistics show that PWD are more deprived to access to health, education, and employment than people without disabilities $(1,18)$. The World Disability Report states that PWD have less access to health care and unmet needs (1). Similar findings have been reported in several countries $(8,19,20)$.

Given the complexity and abstraction of the phenomenon, the lack of knowledge in this area, and its relevance to different communities, this study was conducted to gain a better understanding based on the experiences of different health care and rehabilitation stakeholders. It has also sought to identify barriers to the implementation of Articles 25 and 26 of CRPD and to assist policymakers, planners, and providers in removing these barriers and improving the quality of rehabilitation services, which hopefully ultimately leads to the integration of PWD and improve their quality of life. 


\section{Methods}

Study design

This research was performed using conventional content analysis methodology. In this research, we didn't use any previous hypothesis, and the data were gathered directly based on the participant's experiences. Constant comparative analysis was deployed for data analysis. According to participants' experiences, concepts and codes were summarized and categorized based on differences or similarities, and main categories were developed.

\section{Setting}

The participants were selected for this study in Tehran, who had at least five years of experience in policy-making or implementing programs and activities related to persons with disabilities and a willingness to participate in this research and describe their experiences. Twenty-one people took part in this study. The study setting was the natural environment, and the experiences of participants were investigated at their offices. The purposeful sampling method with the most diversity was used for sampling in interviews with policymakers, managers and heads of non-governmental organizations with different education, experiences and backgrounds in rehabilitation in the pub- lic and private sectors. The demographic characteristics of the participants are presented in Table 1.

\section{Data collection}

Data was gathered by the first author through face to face interviews. The interviewer initially introduced herself and explained the aim of the study and received informed consent from participants. Upon receiving the participants' agreement, the interviewers were recorded. During initial interviews, the researcher asked general and open-ended questions in order to understand participants' perceptions and experiences with barriers to the implementation of articles 25 and 26 of the CRPD in Iran. Interviews also contained probing questions (for example, "Can you give me an example?"' and "Please speak more about it?"'). The follow-up questions were asked according to information provided by the participant to clarify the concept under study. Data collection was continued until saturation. Data were considered saturated when no more codes could be identified and the properties and dimensions of the category were coherent or made sense. The interviews were conducted from June 2018 to May 2019. The time of each interview was between 15 and 50 minutes. All participants took part in one interview.

\begin{tabular}{|c|c|c|c|c|c|c|}
\hline Code & Field & Job & Sex & Education & Sector & Experience (year) \\
\hline 1 & Higher Education Management & Manager & Male & $\mathrm{PhD}$ & Public & 25 \\
\hline 2 & Health Care Management & Manager & Male & $\mathrm{PhD}$ & Public & 20 \\
\hline 3 & Health Education & Policymaker & Male & $\mathrm{PhD}$ & Public & 30 \\
\hline 4 & Occupational Therapy & Expert & Female & MSc & Private & 27 \\
\hline 5 & Psychology & Expert & Male & $\mathrm{PhD}$ & Public & 15 \\
\hline 6 & Graphic & N.G.O & Female & $\mathrm{BSc}$ & Private & 16 \\
\hline 7 & Physiotherapy \&Management & Policy maker & Male & $\mathrm{PhD}$ & Public & 30 \\
\hline 8 & Strategic Management & Policy maker & Male & $\mathrm{PhD}$ & Public & 28 \\
\hline 9 & Elderly Science & Expert & Male & $\mathrm{PhD}$ & Public & 10 \\
\hline 10 & Management & Policy maker & Male & MSc & Public & 27 \\
\hline 11 & Rehabilitation Management & Manager & Male & MSc & Public & 20 \\
\hline 12 & Rehabilitation Management & N.G.O & Male & MSc & Private & 30 \\
\hline 13 & Rehabilitation Management & Manager & Male & MSc & Public & 20 \\
\hline 14 & Rehabilitation Management & Policy maker & Male & MSc & Public & 25 \\
\hline 15 & Speech Therapy & N.G.O & Male & $\mathrm{BSc}$ & Private & 24 \\
\hline 16 & Psychology of Exceptional Children & N.G.O & Male & $\mathrm{PhD}$ & Private & 15 \\
\hline 17 & general surgery & Manager & Male & MD & Public & 18 \\
\hline 18 & Health Care Management & Expert & Male & $\mathrm{PhD}$ & Public & 18 \\
\hline 19 & International Law & Expert & Male & $\mathrm{PhD}$ & Private & 8 \\
\hline 20 & Health and Social Welfare & Expert & Male & $\mathrm{PhD}$ & Public & 12 \\
\hline 21 & Rehabilitation Management & Manager & Male & MSc & Public- Private & 15 \\
\hline
\end{tabular}




\section{Data Analysis}

The data was processed through the content analysis method. Interviews were tape-recorded and transcribed verbatim. Information obtained was repeatedly read to gain a general understanding of it. Then, data were analyzed using Graneheim and Lundman approach (2004); This means data were divided into meaning units, condensed, labeled as codes, constantly compared to develop sub-categories and categories (21). Condensing meaning units was done by process of reducing the text while maintaining their core and was coded accordingly after the process of coding and grouping the codes. Finally, obtaining underlying meanings, they were discussed by the researchers' team and revised as main categories; it should be noted that data gathering and data analysis were conducted in parallel and simultaneously. In each section, with reflection and discussion resulted, peer check was used to reach a consensus between research team members. The MAXQDA software version 10 as well as manually, was utilized in the analysis process.

\section{Trustworthiness}

In the current study, we used Lincoln and Guba's criteria, including credibility, dependability, conformability, and transferability (22). To increase the credibility of the data, it is necessary to be involved within data for a longtime. Therefore, the researchers were involved in data collection for 13 months and using member check with participants in the end of each interview and after the process of coding and participants confirmed the reflected interviews (23). In addition, dependability was assessed by the peer check strategy (24). To enhance conformability, all the background and stages of study, especially the data analysis, were documented. Therefore, other interested researchers had access to these documents to use them in possible future studies (22). For transformability, our research team provided the conditions to use the results in a variety of settings. To facilitate transferability, it is valuable to give a clear and distinct description of culture and context, selection and characteristics of participants, data collection and process of analysis. A rich and vigorous presentation of the findings together with appropriate quotations, will also enhance transferability (21).

\section{Ethical Considerations}

The informed consent was obtained from the participants via clarifying the purpose and the process of the study. This study was adopted from the first author's master's degree thesis and was approved by the Ethical Committee of the University of Social Welfare and Rehabilitation Sciences (USWR) in Tehran, Iran (IR.USWR.REC.1397.105).

\section{Results}

Twenty-one participants (19 men and 2 women) took part in this study. Participants included 5 policymakers, 6 rehabilitation managers, 6 expert in rehabilitation and 4 heads of non-governmental organizations with different education, experiences and backgrounds in the public and private sectors. All of the participants had work experience more than five years in the field of rehabilitation.

The study provided a new perspective on the important barriers to the implementation of Articles 25 and 26 of the International CRPD. The findings in 7 categories and 27 subcategories are presented in Table 2.

Table 1. Barriers to the implementation of articles 25 and 26 of the international CRPD

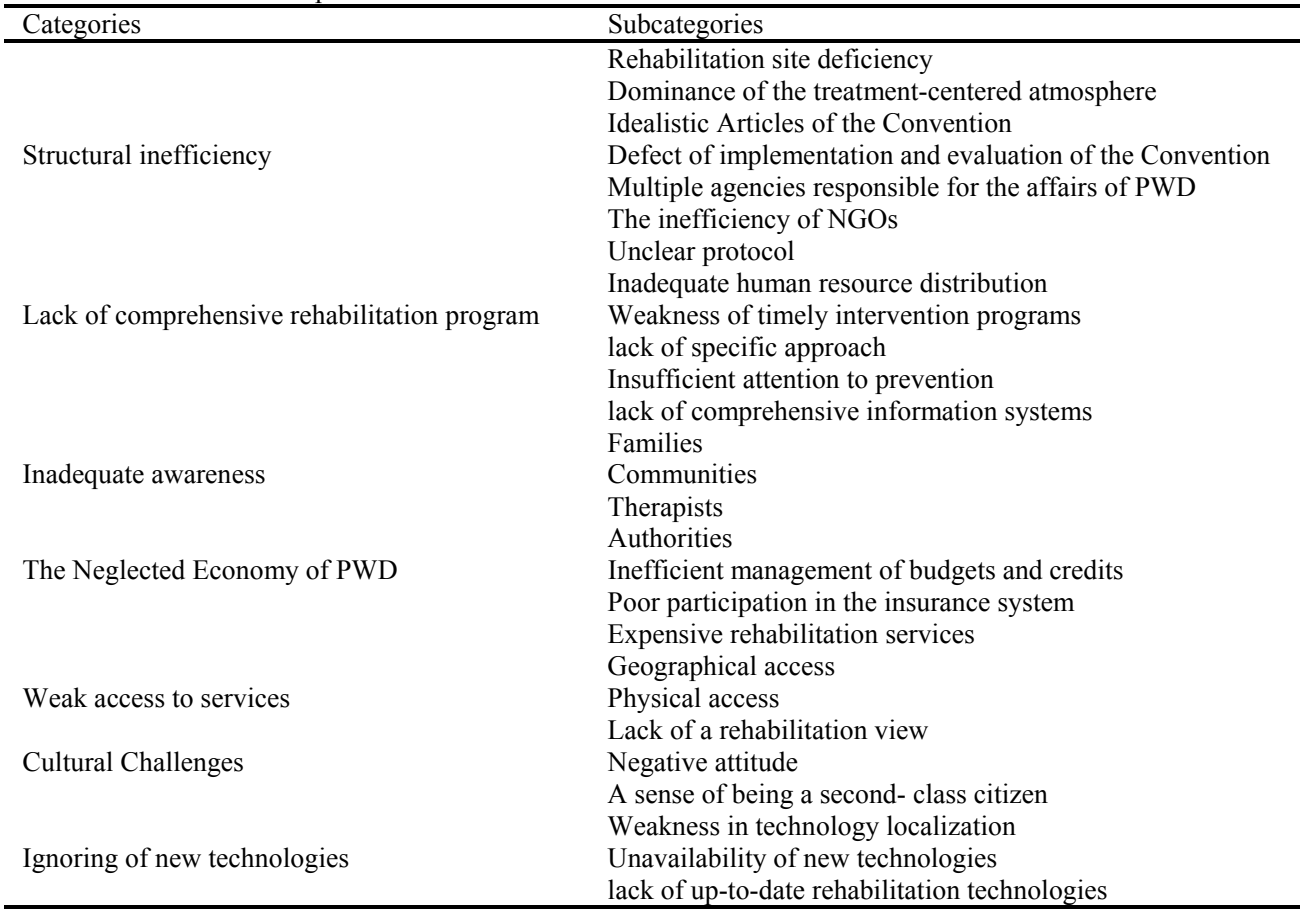




\section{Structural inefficiency}

The findings showed that one of the main challenges and concerns most participants stated was structural inefficiency, which extracted from 6 sub-categories including; "rehabilitation site deficiency", "dominance of the treatment-centered atmosphere", "idealistic Articles of the Convention", "defect of implementation and evaluation of the Convention", "multiple agencies responsible for the affairs of PWD", and "the inefficiency of NGOs. The last one is the most important requirements for implementing health and rehabilitation materials that, if inadequate, can disrupt the implementation of the whole programs and laws.

A contributor to Rehabilitation site deficiency stated:

"Rehabilitation is a subset of the ministry of Health that needs to be separated. Even in the upstream documents, rehabilitation is under the shadow of the Ministry of Health, and we do not have an upstream policy document to implement the rehabilitation services. On the other hand, unfortunately, the Cooperatives have not yet reached a clear understanding of the division of responsibilities in the field of rehabilitation". (Participant19)

\section{Lack of a comprehensive rehabilitation program}

Many participants pointed out that there is no comprehensive rehabilitation program in our country that raises other issues such as disruption of performance, parallelism, and so on. This category comprises six sub-categories of "unclear protocol", "inadequate human resource distribution", "weakness of timely intervention programs", "lack of specific approach", "insufficient attention to prevention", and "lack of comprehensive information systems". Another participant noted:

"We do not have a comprehensive rehabilitation program in our country to exactly clarify the goals and policies for PWD. A part of this program is the responsibility of the Ministries, and another part is related to the municipalities and in general, we have no plans for rehabilitation services. In relation to the rehabilitation, even the deputy director of rehabilitation has a number of stereotyped strategies, and they did not meet to formulate a plan of action with the help of stakeholders, their different work partners, and different people involved in a comprehensive plan and strategy". (Participant 4)

Another Participant about prevention stated:

"No one has ever told people about prevention. The health care system is ruled in the country. It seems to say, "Let's build a prison". Well, it means we build to fill it! However, if society thinks about prevention, it does not think about prison. In a community, if health is respected and prevention is at the forefront, fewer people will need treatment and hospital-care". (Participant 3)

Another Participant regarding the lack of a comprehensive information system said:

"One of the problems that we have required to implement Articles 25 and 26 is the lack of a strong mechanism for information. How much of the statistics that we use in the census to provide the facilities to PWD are useful? Their statistics are imperfect. If you want to census, we need these items. However, they say we cost millions of dollars for every question we want to put in the census. If we want to have proper planning, we need to work on good statistics in terms of the number of PWD, the types of needs, and the number of people who are regularly added.". (Participant 10)

\section{Inadequate knowledge}

One of the major issues that most of the participants pointed out was the inadequate knowledge of the families, communities, therapists, and authorities, which emphasized the need to pay attention to this subject. Unfortunately, the lack of awareness about PWD is one of the major issues and there are many problems to address. In this regard, one participant noted:

"Another obstacle is that our knowledge is limited; that is to say, education and knowledge cannot be injected into society to soothe the eyes, but education must take shape from childhood and kindergarten. As those children enter school and then to university and become a manager, a responsible, and/or a minister, their mind would be practically trained. Therefore, lack of awareness and training for the community is a barrier that we necessarily seek to address". (Participant 8)

\section{The Neglected Economy of PWD}

This category was one of the main barriers to the implementation of the CRPD in Iran. During the interviews, all participants identified this as one of the most important concerns that play a prominent role in receiving and delivering rehabilitation and health services. It has challenged service delivery and, in most cases, increased the severity of disability due to high costs and taking a long time. It also overshadowed the provision of rehabilitation services and resulted in interruption or non-follow-up of rehabilitation. It extracted from the following subcategories: "inefficient management of budgets and credits", "poor participation of the insurance system", and "expensive rehabilitation services". One interviewee described the obstacle as:

"Another important issue is financing, which means that if we want to have a nationwide program for all groups, we need to be able to predict a credit line. There is no credit line in the Ministry of Health because the insurance system is in trouble. Every disabled person's per capita income in the Welfare Organization is 900,000 Toomans a year, so how can it be provided with jobs, housing, rehabilitation services, and education? This causes a lot of need and low-income results in low costs". (Participant 2)

Another contributor explained:

"The Welfare Organization reduces the insurance budget each year, while the needs of the service seekers are very high. The organization is not able to respond because the health budget of the country is given to the Ministry of Health and parallelism in work has caused a loss of budget." (Participant 4)

\section{Weak access to services}

Access to services is one of the main concerns of participants in providing and receiving rehabilitation and health 
services, most of which were mentioned in interviews and were considered as a prerequisite for providing rehabilitation services. This category extracted from subcategories Included; the "geographical access" and "physical access" sub-categories. One of the participants said:

"We do not have suitable access to health services in our different parts of the country. We now observe health homes in all rural areas that provide primary health care, but we have a lot of difficulties in getting second and third-level services. That is to say, PWD had to travel far if they want to come to a town from the village, but there was only one doctor in town while one needs a specialist who did not exist. So, inaccessibility in terms of geographical barriers makes the quality and effectiveness of treatment difficult and impedes the realization of the Article. In terms of construction, we do not see a medical or dental health center for PWD at the Ministry of Health, while dentistry for them is a specialized job". (Participant 2)

\section{Cultural Challenges}

One category that is a major obstacle to the presence of PWD in the community and access to the services they need is the cultural barriers existing among the community, officials, therapists, families, and even PWD themselves. This category has three basic sub-categories, including "lack of a rehabilitation view", "negative attitude", and "a sense of being a second-class citizen". Relating to a negative attitude, a contributor stated:

"The biggest challenge is the lack of a "positive attitude". They consider PWD as a few numbers of people. They do not really care that reviving a human being is a revival of society as a whole. The idea of "revival" in the Qur'an does not mean that you just bring life to other people, but that one must come into the community and be able to live and participate. This attitude prevents serious attention to persons with disabilities. They focus more on the disabilities of such persons, while they are, in fact very capable people in doing their jobs. In the world, we see a minister who is blind, but we have obstacles in our law at present that prevent PWD from to enter into the parliament. This is a challenge that actually has the negative attitude that we face even in the law". (Participant7)

Another contributor regarding "being a second-class citizen," stated:

"They come to the community with the stigma and people lookat them as different. Although many things changed compared with a decade ago, it is still the same; that is, ordinary people look at PWD as second-class citizens". (Participant9)

\section{Ignoring new technologies}

New technologies of rehabilitation will undoubtedly play an obvious and undeniable role in better adapting to existing constraints, achieving greater independence, and ultimately reducing the burden of care for families. One of the barriers, considered in the present study, is the ignoring of new technologies, which include the three basic sub-categories of "weakness in technology localization", "lack of up-to-date rehabilitation technologies", and "unavailability of new technologies". In this regard, one partic- ipant described:

"How much have we been able to access technology tailored to our disability? Wheelchairs, as the simplest device, are usually just in one type, and people with different disabilities and different height and weight characteristics all use the same type. The conditions are more difficult in sophisticated technologies such as hearing aids. In addition, technologies that can make their working environment comfortable, such as design a special pen, ergonomics of the workplace, etc., are the major obstacles to the realization of these two Articles". (Participant 11)

\section{Discussion}

This study was conducted because of the importance of providing rehabilitation and health services for PWD. They showed various factors prevent the implementation of the International CRPD in Iran. In this study, based on the participants' experience and understanding, the main barriers included "inefficiency", "lack of comprehensive rehabilitation program", "inadequate knowledge", "neglected economy of PWD", "limited access to services", " cultural challenges", and "ignoring new technologies". Other studies have addressed some of these barriers, which are discussed below.

One of the major barriers to the implementation of the Articles of the Health and Rehabilitation Convention was the inefficiency of the structure, which included the subcategories of "rehabilitation site failure", "treatmentcentered dominance", "idealistic Articles of the Convention", "failure to implement and evaluate the Convention", "multiple centers in charge of people with disability", and "the inefficiency of NGOs".

The study by Abdi et al. (2016), pointed "multiple responsible centers", "the unknown situation of rehabilitation", and "hospital-centered hospital atmosphere", which confirms the findings of our study (25). Dalvandi's study also showed not health cause functional impairment (26). In the study of Soltani et al., the importance of the associations and NGOs related to PWD has been underscored, which confirms the findings of the present study (27). Besides, our study also provided new findings, including the idealistic Articles of the Convention and the inefficiency of NGOs that are major barriers to rehabilitation services for PWD.

One of the most important challenges in implementing Article 26 of the Convention, namely rehabilitation, was the deficiency of a comprehensive rehabilitation program that has six major sub-categories, including "unclear protocol", "inadequate human resource distribution", "weakness of timely intervention programs", "lack of a clear approach", "inadequate attention to prevention" and "lack of comprehensive information systems".

According to the WHO report, in 2005, the UN found standard rules on equal opportunities for PWD conducted a global survey of non-binding implementation. In 42 percent of participating countries, no rehabilitation policies were adopted; in 50 percent of countries, the Rehabilitation Act for Persons with Disabilities was not enacted, and in 40 percent, there were no statutory rehabilitation programs. Many countries had appropriate laws and policies 
related to rehabilitation but wrapped behind in implementing these policies, developing, and delivering local and regional rehabilitation services (1).

In the present study, also lack a program one of sub-categories extracted in the interviews of the participants that was a major obstacle to the provision of rehabilitation services in country. The superiority of our study compared to the aforementioned survey was the introduction of new sub-categories, including the "weakness of timely intervention programs," "inadequate attention to prevention," "and the lack of a comprehensive information system".

The study by Eldar et al. (2008), on physical rehabilitation of physical disorders, showed that assistive technology, sensory trauma, and specific procedures that could lead to unequal distribution of services (28). In addition to the lack of a strategic plan, our study also found cases of "inadequate human resource distribution", "weakness of timely intervention programs", "inadequate attention to prevention", and "lack of comprehensive information systems".

One of the categories extracted from the interviews was "insufficient knowledge", which includes four subcategories. The subcategories demonstrate the need to foster knowledge at all levels, including "family", "community", "therapists", and most importantly "authorities" in the field.

According to the findings of Andrew Long et al. (2003), insufficient understanding and skill of nurses is related to educational programs and the gap between nurses' educational understanding and effective skill and knowledge for working in rehabilitation settings (29).

In a study by Bahareh Rezaei Nick (2017), one of the major barriers to cooperation among nurses was poor educational planning (30). In another study by Abdi et al. (2015), one of the major concerns of the majority of participants, especially managers, policy-makers, and providers of rehabilitation services in the academic rehabilitation system, was that it might cause insufficient awareness of postgraduate service providers in providing rehabilitation services (11). A study by Hosseini et al. (2010) assessed the level of awareness of the clients of rehabilitation centers about the provisions of the patients' rights; they emphasized that there was awareness, education, the frequency of visits, and the rehabilitation ward. The study reported that the knowledge of the therapists at the rehabilitation centers was $53.6 \%$ good, $32.1 \%$ poor, and $14.3 \%$ moderate (31), which is consistent with the findings of our study.

Among the very important categories that the majority of participants in the present study referred to were the "disregarded economy of PWD". It has three subcategories of "inefficient budget and credit management", "poor insurance system participation", and "expensive rehabilitation services"; they are considered as the major and fundamental barriers to promoting the rights of PWD and access to desirable rehabilitation and health services. In studies by Megan Hussey et al. and Abdi et al., the results indicated that the "financial" barrier is the main barriers to providing rehabilitation services, which are con- sistent with the findings of our study $(6,11)$. In Lee Jung's study (2014), 58.8\% of PWD in Korea stated that they did not receive medical services when they needed because they have no money, which is a very high percentage and confirmed the results of the present study (32). Also in the study of Abasi (2009), the most economical problems of families with mentally retarded children were in three areas of medical and pharmaceutical costs $(30.7 \%$, ) food and clothing (24.3\%), and transportation costs $(23.6 \%)$, respectively. These problems were because of incomplete coverage of health insurance. Likewise, low family income, lack of a suitable job, and lack of education to obtain a job can intensify these problems (33). In the research by Jahanbin et al. (2019), lack of financial resources has been mentioned as one of the major problems in the field of rehabilitation (34). The findings of all these studies confirm the findings of the present study suggesting inadequate insurance coverage, high cost of rehabilitation services, and other financial problems.

As noted in the findings, access to services was one of the influential factors in providing and receiving a rehabilitation and health service that was a major concern of our research participants, and mentioned by most of the interviewees, and is considered as a prerequisite to providing rehabilitation services. Access includes the "geographical access" and "physical access" sub-categories. Murthy Venkata (2014) in Southern India PWD faced more barriers to accessing health services than people without disabilities. Obstacles included: "ignoring service availability," "service costs," and "transportation problems", which was consistent with our research findings. Obstacles included: ignoring service availability, service costs, and transportation problems (35), which was consistent with our research findings. In another study by Mégan Hussey (2015), explaining the barriers to the implementation of health and rehabilitation materials at the international CRPD in South Africa, the findings emphasized the existence of physical barriers to health and rehabilitation (6) which is fully consistent with the findings of our study. The advantage of the present study is the addition of "geographical accessibility" to these services.

One of the categories that has become a major barrier to PWD in the community and accessing the services they need was the "cultural barriers" at the community level, officials, therapists, families, and even PWD, and this category has three basic sub-categories including "lack of a rehabilitation view", "negative attitude", and "a sense of being a second class citizen". A study by Megan Hussey et al., as well as a qualitative study by Maria Zoormand et al. (2019) entitled "Barriers and Facilitators of access to health services for PWD in Cameroon and India" address the barriers at the level of attitude of the family and community of the persons with disabilities $(6,36)$, which corroborates the findings of the present study. Moreover, the present study also emphasized the "weaknesses of the authorities" and "second-class citizen", which was neglected in previous studies.

"New technologies of rehabilitation", undoubtedly, play an obvious and undeniable role in better adapting to existing constraints, achieving greater independence, and 
ultimately reducing the burden of care for the families and other caretakers. One of the barriers innovated by the present study, which was overlooked in previous studies, is "disregard for new technologies", which includes the three basic sub-categories of "weakness in technology localization", "lack of up-to-date rehabilitation technologies", and "unavailability of the new technologies". Only a few studies, such as those of Khanjani et al., have addressed the importance of rehabilitation assistive technologies (37).

\section{Limitations}

In the present study research team did not interview with PWD and her families, it is recommended to pay more attention to it in the future studies.

\section{Conclusion}

This study has been explored barriers to the implementation of articles 25 and 26 of the CRPD in Iran. It identified challenges and the concerns for all rehabilitation team and health services, policy-makers and managers, therapists, and NGOs that service to the PWD. In the next step, it can be helpful in addressing or eliminating the challenges identified, and ultimately developing a comprehensive plan. The findings showed that the executive structure in country have problems with health and rehabilitation programs for PWD. On the one hand, there is a lack of a comprehensive rehabilitation program to present a specific protocol that should be able to address, with adequate and timely attention to prevention and interventions, a large part of the challenges existing in achieving the rights of persons with disabilities in Iranian health system. Nevertheless, it is also necessary that policymakers they must change their insight to rehabilitation services.

\section{Acknowledgment}

The authors thank all participants in this research.

\section{Conflict of Interests}

The authors declare that they have no competing interests.

\section{References}

1. organization wh. World report on disability 2011 [Available from: https://www.who.int/disabilities/world_report/2011/en/.

2.Akhavan-TaftiM. CRPD: A Review of Challenges and Gaps in Its Implementation in Some Countries. J Res Rehabil Sci. 2016;12(3):173-84.

3. Tavasoli M, Kaviar M. Reflection on the Rights of Persons with Disabilities from the Perspective of the CRPD and Other International Documents. Private law. 2008;5(13):29-50.

4. Lang R, Kett M, Groce N, Trani JF. Implementing the United Nations CRPD: principles, implications, practice and limitations. Alter. 2011;5(3):206-20.

5. United Nation convention on the right of persons with disabilities (CRPD) 2006 [Available from: https://www.un.org/development/ desa/disabilities/convention-on-the-rights-of-persons-with-disabil ities.html.

6.Hussey M, MacLachlan M, Mji G. Barriers to the Implementation of the Health and Rehabilitation Articles of the United Nations CRPD in South Africa. Int J Health Policy Manag. 2016;6(4):207-18.

7. Kim HS. UN disability rights convention and implications for social work practice. Aust Soc Work. 2010;63(1):103-16.
8. Shin J, Moon S. Quality of care and role of health insurance among non-elderly women with disabilities. Womens Health Issues. 2008;18(4):238-48

9.B jarnason-Wehrens B, Grande G, Loewel H, Voller H, Mittag O. Gender-specific issues in cardiac rehabilitation: do women with ischaemic heart disease need specially tailored programmes? Eur J Cardiovasc Prev Rehabil. 2007;14(2):163-71.

10. Petrea RE, Beiser AS, Seshadri S, Kelly-Hayes M, Kase CS, Wolf PA. Gender differences in stroke incidence and poststroke disability in the Framingham heart study. Stroke. 2009;40(4):1032-7.

11. Abdi K, Arab M, Rashidian A, Kamali M, Khankeh HR, Farahani FK. Exploring barriers of the health system to rehabilitation services for PWD in Iran: A qualitative study. Electron Physician. 2015;7(7):1476.

12. Agah V. Labour law for persons with disability in Iran: From first international efforts to decent work. Arch Rehabil. 2012;13(1):88101.

13. Nouri A. Disability Rights Charter. Office of Culture for the Disabled, 2015, Ghom

14. Nasib SM, Jahanbin SE, Jahanbin SF. An Overview of the Legal Status of Disabled Persons in Iran. J Fund Rights. 2011;16.

15. Nastaran M. Analysis and measuring degree of concentration and distribution of health indices in Isfahan. $J$ Fac Lit Hum. 2001;27(1):145-62

16. Abolhallaje M, Mousavi SM, Anjomshoa M, Nasiri AB, Seyedin $\mathrm{H}$, Sadeghifar J, et al. Assessing health inequalities in Iran: a focus on the distribution of health care facilities. Glob J Health Sci. 2014;6(4):285.

17. Ahmadi A, Yousefi M, Fazayeli S. Analyze changes in inflation, public sector and health sector of the country. J Econ Res. 2010;10(1):99-111.

18. Najafi Z, Abdi K, Khanjani MS. Disability and the Convention on the Rights of Persons with Disabilities in Iran: What Can We Do? J Rehabil Sci Res. 2019;6(2):101-2.

19. Maart S, Jelsma J. Disability and access to health care-a community based descriptive study. Disabil Rehabil. 2014;36(18):1489-93.

20. Sommers AS. Access to health insurance, barriers to care, and service use among adults with disabilities. Inquiry. 2006;43(4):393405.

21. Graneheim UH, Lundman BJNet. Qualitative content analysis in nursing research: concepts, procedures and measures to achieve trustworthiness. Nurse Educ Today. 2004;24(2):105-12.

22. Guba EG. Criteria for assessing the trustworthiness of naturalistic inquiries. Int J STEM Educ. 1981;29(2):75-91.

23. Beck CT. Qualitative research: The evaluation of its credibility, fittingness, and auditability. West J Nurs Res. 1993;15(2):263-6.

24. Lincoln YS. Emerging criteria for quality in qualitative and interpretive research. Qual Inq. 1995;1(3):275- 89.

25. Abdi K, Arab M, Khankeh HR, Kamali M, Rashidian A, Farahani FK, et al. Challenges in Providing Rehabilitation Services for PWD in Iran: A Qualitative Study. Br J Med Med Res. 2016;13(4):1-11.

26. Dalvandi A, Heikkilä K, Maddah S, Khankeh H, Ekman SL. Life experiences after stroke among Iranian stroke survivors. Int Nurs Rev. 2010;57(2):247-53.

27. Soltani S, Takian A, Sari AA, Kamali M, Majdzadeh SR, Matin BK. Disregarded Health Problems of PWD: A Qualitative Study of Policymakers' Perspective. Arch Rehabil. 2019;20(2):136-48.

28. Eldar R, Kullmann L, Marincek C, Sekelj-Kauzlarić K, Švestkova $\mathrm{O}$, Palat M. Rehabilitation medicine in countries of central/eastern Europe. Disabil Rehabil. 2008;30(2):134-41.

29. Long AF, Kneafsey R, Ryan J. Rehabilitation practice: challenges to effective team working. Int J Nurs Stud. 2003;40(6):663-73.

30. Rezaee NB, Rasouli M, Vaskoei Ek, Alavi MH, Torabi F. The obstacles and facilitating of nurses collaborative in clinical education nursing student. J Nurs Educ. 2017;6(5):62-8

31. Hosseini SA, Akbar-Fahimi N, Gharib M, Farzad M. Therapist and Client Awareness of Client's Rights. Arch Rehabil. 2013;13(4):28-36

32. Lee JE, Kim HR, Shin HI. Accessibility of medical services for persons with disabilities: comparison with the general population in Korea. Disabil Rehabil. 2014;36(20):1728-34

33. Abasi S, Fadakar K, Khaleghdoost T, Sedighi A, Atrkar Roshan Z. Survey the problems of families with mentally retarded child covered by Guilan Welfare Centers in2010. J Holist Nurs Midwif. 
2010;20(1):33-9.

34. Jahanbin P, Abdi K, Khanjani MS, Hosseini MA. Exploring barriers of teamwork in providing rehabilitation services: A Qualitative content analysis. Arch Rehabil. 2019;20(3):210-21.

35. Gudlavalleti MVS, John N, Allagh K, Sagar J, Kamalakannan S, Ramachandra SS. Access to health care and employment status of PWD in South India, the SIDE (South India Disability Evidence) study. BMC Public Health. 2014;14(1):1125.

36. Zuurmond M, Mactaggart I, Kannuri N, Murthy G, Oye JE, Polack S. Barriers and Facilitators to Accessing Health Services: A Qualitative Study Amongst PWD in Cameroon and India. Int J Environ Res Public Health. 2019;16(7):1126.

37. Khanjani MS, Khankeh HR, Younesi SJ, Azkhosh M. The Main Factors Affecting the Acceptance and Adaptation With Spinal Cord Injury: A Qualitative Study. Arch Rehabil. 2019;19(4):276-91. 